\title{
Histopathology of a benign bile duct lesion in the liver: Morphologic mimicker or precursor of intrahepatic cholangiocarcinoma
}

\author{
Kyoung-Bun Lee \\ Department of Pathology, Seoul National University Hospital, Seoul, Korea
}

\begin{abstract}
A bile duct lesion originating from intrahepatic bile ducts is generally regarded as an incidental pathologic finding in liver specimens. However, a recent study on the molecular classification of intrahepatic cholangiocarcinoma has focused on the heterogeneity of this carcinoma and has suggested that the cells of different origins present in the biliary tree may have a major role in the mechanism of oncogenesis. In this review, benign intrahepatic bile duct lesions-regarded in the past as reactive changes or remnant developmental anomalies and now noted to have potential for developing precursor lesions of intrahepatic cholangiocarcinoma-are discussed by focusing on the histopathologic features and its implications in clinical practice. (Clin Mol Hepatol 2016;22:400-405)
\end{abstract}

Keywords: Intrahepatic cholangiocarcinoma; Ductal plate malformation; Bile duct adenoma; Peribiliary cyst

\section{INTRODUCTION}

Recently, the heterogeneity of intrahepatic cholangiocarcinomas (ICCS) with regard to its histopathogenesis and molecular alteration has been revealed, and this heterogeneity may reflect the diverse histophysiology of cholangiocytes present along the biliary tree.' ICC can be divided into the peripheral small duct type and perihilar large duct type. ${ }^{2}$ The peripheral small duct type ICC is considered to originate from the interlobular small bile duct and mass-forming ICCs are included in this type. The perihilar large duct type ICC has a similar phenotype with cholangiocarcinoma of the extrahepatic bile duct or hilar cholangiocarcinoma (so called "Klatskin tumor"), and the periductal infiltrative type or intraductal growing type of ICC are included in this group. The basic concept of precursor lesions of large duct type ICC is similar to the general concepts of "dysplasia-carcinoma sequence" or "adenoma-carcinoma sequence" that elucidate carcinogenesis in the gastrointestinal tract; this is because the comprising epithelial cells are mucinous epithelial cells similar to those in the gastrointestinal tract. However, peripheral small duct type ICC has a different histologic phenotype, which is the non-mucinous type, which shows homology or transitions to hepatocellular carcinoma (HCC). Amid growing interest in precursor lesions of peripheral small duct type ICCs, benign intrahepatic bile duct lesions, which have been regarded as reactive changes or remnant developmental anomalies in the past, have been noted to have potential for developing precursor lesions.

This paper reviewed the histopathology of benign bile duct le-

\section{Abbreviations:}

ADPKD, autosomal dominant polycystic kidney disease; DPM, ductal plate malformation; HCC, hepatocellular carcinoma; ICC, intrahepatic cholangiocarcinoma; PCLD, isolated polycystic liver disease; PLD, polycystic liver disease; RFA, radiofrequency ablation; TACE, transarterial chemoembolization; VMC, von Meyenburg complexes

\section{Corresponding author: Kyoung-Bun Lee}

Department of Pathology, Seoul National University Hospital, 101 Daehak-ro, Jongno-gu, Seoul 03080, Korea

Tel: +82-2-2072-2968, Fax: +82-2-743-5530

E-mail: kblee@snuh.org 
sions and their clinicopathologic implications and biologic relevance to ICC.

\section{NORMAL HISTOLOGY OF THE BILIARY TREE AND DEVELOPMENT}

The biliary tract in terminal portal tracts is lined by a single layer of cuboidal non-mucinous epithelial cells, which changes to mucinous tall columnar epithelial cells in septal or hilar bile ducts that which have distinct walls composed of fibromuscular stroma and peribiliary glands. Bile ducts in the terminal portal tract are developed from ductal plates originating from primitive hepato- cytes surrounding the portal vein; therefore, they have a dual phenotype of cholangiocytes and hepatocytes at an early period. ${ }^{3}$ After remodeling and reabsorption of an anastomosing irregular ductal plate, bile ducts in the terminal portal tract have a single lumen and a physiologic continuum with the canaliculi of hepatocytes, and thus make a protective transportation system for bile. On the contrary, extrahepatic biliary systems arise from the ventral endoderm of the foregut and continue with the duodenal epithelium at the caudal end and with primitive a hepatic sheet at the cephalic end. ${ }^{3}$ The intrahepatic large bile duct can be considered as an intrahepatic extension of the extrahepatic bile duct system and may have similar histologic features with the extrahepatic bile duct. The precise anatomic separation point between
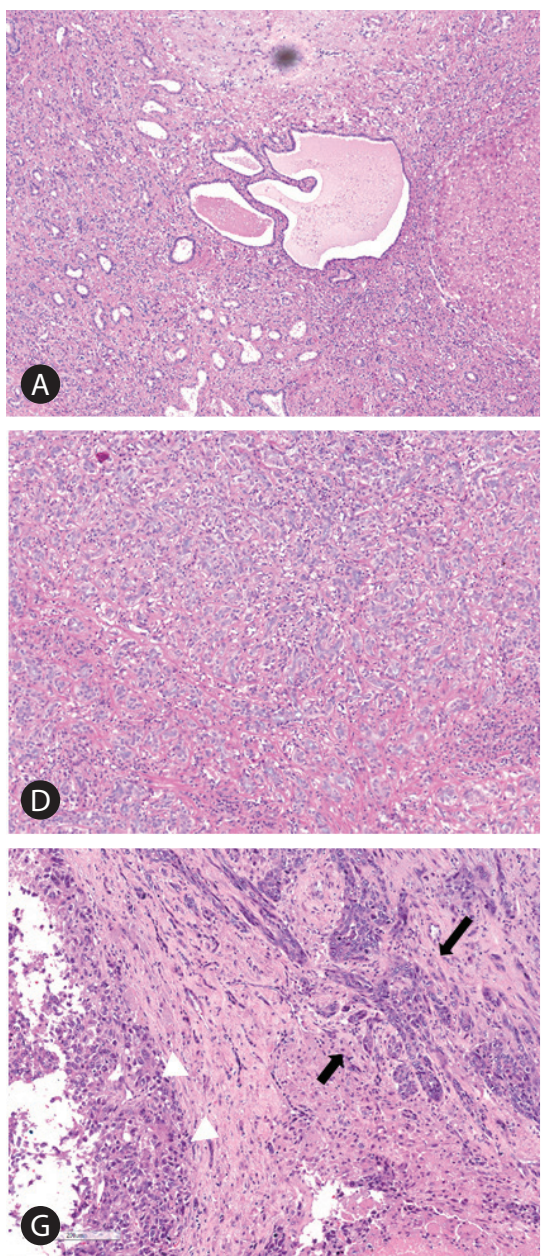
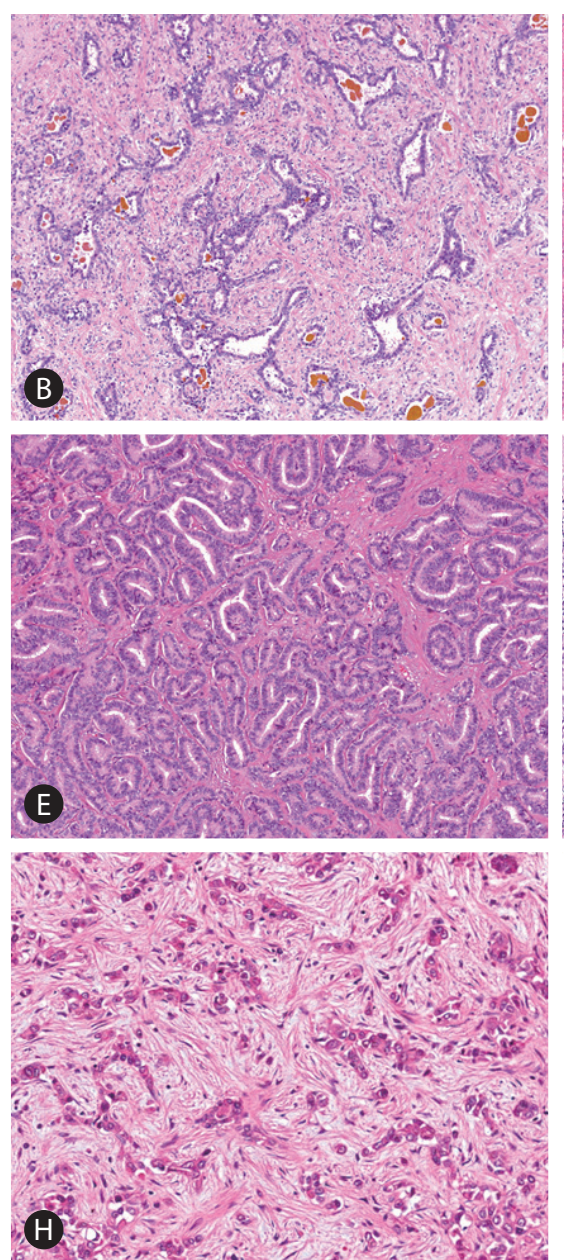
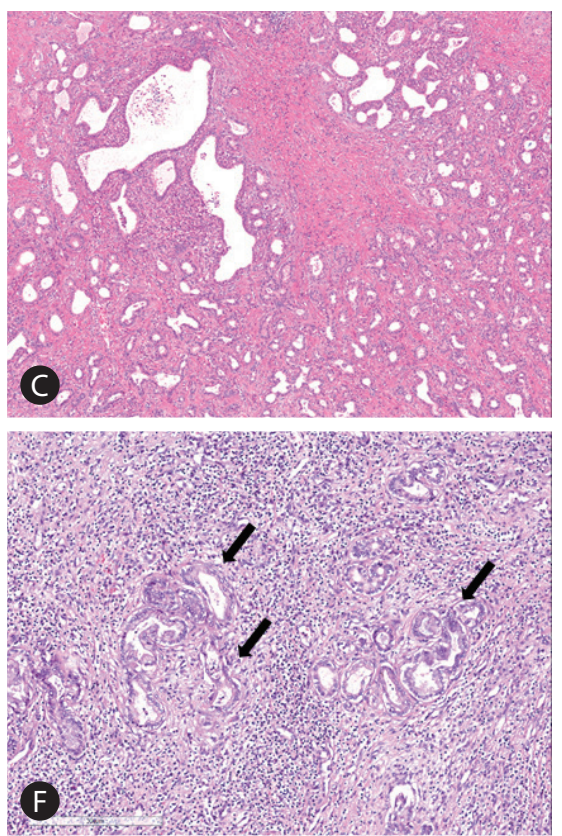

Figure 1. Benign bile duct lesion and mimicking intrahepatic cholangiocarcinoma. (A) Von Meyenburg complex (VMC) composed of irregularly dilated small bile ducts (HE stain, $\times 66$ ). (B) Prominent VMCs with inspissated bile juice in lumen (HE stain, $\times 84$ ]. (C) Ductal plate malformation like intrahepatic cholangiocarcinoma ( $H E, \times 50)$. (D) Bile duct adenoma composed of small packed glands (HE stain, $\times 100)$. (E) Well differentiated intrahepatic cholangiocarcinoma mimicking bile duct adenoma (HE stain, $\times 100$ ). (F) Atypical ductular reaction (arrows) around regenerating nodule of Hepatitis B Virus-associated cirrhosis (HE stain, $\times 100$ ] (G) Atypical ductular reaction (arrows) around hepatocellular carcinoma (white arrowhead) pretreated by TACE (HE stain, $\times 100$ ). (H) Cholangiolocellular intrahepatic cholangiocarcinoma (HE stain, X200). TACE, transarterial chemoembolization. 
the intrahepatic large bile duct and intrahepatic small bile duct are not clear. The right and left hepatic duct of the common hepatic duct and the first to third branches (segmental branches) are usually called the intrahepatic large bile ducts and can be grossly visible and are composed of tall columnar mucinous epithelial cells with a collagenous wall. The intrahepatic small bile duct has two levels; i.e., the septal and interlobular bile ducts, which are grossly invisible and the interlobular bile ducts are composed of non-mucinous cuboidal cells and are located in the terminal portal tract. The septal duct has an ambiguous phenotype. It cannot be visualize grossly and is lined by tall columnar epithelial cells surrounding a fibrous duct wall similar to that in the intrahepatic large bile ducts. Peribiliary glands also have similar ambiguous biologic features. Peribiliary glands are usually identified in the intrahepatic large bile duct or extrahepatic bile duct and are described as intramural or extramural according to the location in the bile duct wall. Intramural peribiliary glands arise from the ductal plate similar to the intrahepatic small bile duct, but some extramural peribiliary glands show homology of pancreatic acini, suggesting a common developmental origin with the extrahepatic bile duct orgin. ${ }^{4,5}$

\section{DUCTAL PLATE MALFORMATION AND CYSTIC LIVER DISEASE}

Abnormal arrest of ductal plate remodeling during biliary tree development is called a ductal plate malformation (DPM) and polycystic liver disease (PLD) is the representative entity resulting from a DPM. PLD has three component entities: 1) Von Meyenburg complexes (VMCs), small nonhereditary nodular cystic lesions, 2) isolated polycystic liver disease (PCLD), and 3) autosomal dominant polycystic kidney disease (ADPKD) with multiple cysts in both kidney and liver. PCLD and ADPKD have a genetic background that explains the inheritance pattern. Related genetic alterations are polycystic kidney disease (PKD)1 and PKD2 mutations in ADPKD and protein kinase $\mathrm{C}$ substrate $80 \mathrm{~K}-\mathrm{H}$ (PRKCSH) or the SEC63 mutation in 20\% of PCLD cases. Disrupted TGF- $\beta$, Notch, and Wnt signaling or ciliopathy induce cystic changes of the bile ducts and arrest of DPM. ${ }^{6}$ PCLD and ADPKD show multiple large cysts replacing normal hepatic parenchyma and thus hepatic failure, and complications associated with cysts, such as rupture, hemorrhage or infection, are frequent. These diseases are not risk factors for ICC in general. VMC is a sporadic DPM lesion and is called a biliary microhamartoma; it is easily identified in the adult liver, but not in the pediatric liver, and is usually so small $(<0.5 \mathrm{~cm})$ that it needs to be identified with microscopy. However, some cases showed multiple cysts, which are grossly visible and diffusely located along the biliary tree. Histologically, VMCs are made of irregularly dilated small bile ducts that are embedded fibrous stroma and sometimes have inspissated bile juice in the lumen (Fig. 1A). VMCs and solitary nonparasitic bile duct cysts are considered as part of the spectrum of adult polycystic liver disease, but have no known genetic background or molecular alteration such as ADPKD or PCLD. ${ }^{7}$ The potential role of VMC as a preneoplastic lesion for ICC are based on several studies that have reported ICC arising from VMCs, suggesting malignant transformation from VMC to ICC, ICC having histopathologic similarities with VMC or DPM, and histologic similarity of ICC and VMC in K-ras and p53 mutated animal models inducing ICC. ${ }^{8-10}$ Without large-scale epidemiologic data that can support the relationship between VMC and ICC development, just histopathologic similarity or transition of VMC to ICC cannot be confirmative evidence for VMC as a precursor lesion of ICC. Histologic overlap of VMC and ICC makes it difficult for pathologists to differentiate VMC and ICC, especially for diffuse type VMC (Fig. 1B, C).

\section{BILE DUCT ADENOMA AND DUCTULAR REACTION}

Bile duct adenoma is called a peribiliary gland hamartoma and is composed of small, uniformly sized bile ducts with single cuboidal cells, which look like ductular reactions around regenerating nodules of chronic liver disease. It is small $(<1 \mathrm{~cm})$ and indiscernible in gross examination and detectable in microscopic examination. Compared with VMCs, which are composed of irregularly dilated bile ducts embedded in fibrous stroma, bile duct adenoma has closely packed small glands and the intervening stroma is thinner than that in VMCs (Fig. 1D). Bile duct adenoma was generally regarded as a reactive process to hepatic parenchymal injury; however, a few reports on ICCs arising in bile duct adenoma or malignant transformation of bile duct adenoma to ICC have suggested the possibility of precursor lesions of peripheral ICC (Fig. 1E). ${ }^{11,12}$ A ductular reaction, which looks like a bile duct adenoma, is a common finding in chronic liver disease such as viral hepatitis. A ductular reaction adjacent to HCC is not a common finding, but pretreated HCCs with transarterial chemoembolization (TACE) or radiofrequency ablation (RFA) can show 
exaggerated ductular reactions around tumors with progressing necrosis and fibrosis of the tumor; further, some cholangiocytes have revealed nuclear atypia suggesting neoplastic changes, although these changes are not enough to support a diagnosis of malignancy (Fig. 1F, G). The cholangiolocellular subtype of ICC also has morphologic homology with physiologic ductular reactions in chronic liver disease (Fig. 1H). This entity was first described as a subtype of ICC, but was reclassified as a subtype of combined hepatocellular and cholangiocarcinoma with stem cell features, considering the histopathologic similarity of hepatic progenitor cells in the canal of Hering, which are involved in liver regeneration. ${ }^{13}$ Bile duct adenomas or ductular reactions in normal livers without chronic liver disease can be confused with metastatic adenocarcinoma in the liver. Especially, a frozen examination, performed intraoperatively, can show exaggerated cellular atypia and pathologists should consider the diagnosis carefully. Last, biliary adenofibroma is described as a benign bile duct lesion in the 2010 WHO classification of biliary tumors. ${ }^{13}$ Biliary adenofibroma was first described in 1993 and is so rare that only eight case reports have been published. ${ }^{14}$ Biliary adenofibroma has tubulocytic bile duct proliferation with fibrous stroma. Histologic features are similar to that of VMC apart from its presentation as a localized large mass, while VMC appears as small multiple cystic lesions dispersed in hepatic lobes. Biliary adenofibroma is known to have an indolent clinical course but rates of malignant transformation or coexistence with ICC are relatively high considering the rarity of this entity. ${ }^{15}$ Considering the wide range of morphologic diversity in the bile duct epithelium, it is still controversial whether biliary adenofibroma is a rare variant of extremely well-differentiated ICC or a biliary precursor lesion with high risk of malignant transformation.

\section{PERIBILIARY CYSTS}

Peribiliary cysts were first described in 1984 and it was determined they resulted from cystic dilatation of the intrahepatic extramural peribiliary glands. ${ }^{16}$ Peribiliary cysts are located in the perihilar area and are generally found in chronic liver disease patients, such as liver cirrhosis, alcoholic liver disease, portal hypertension, or thrombosis. ${ }^{17}$ This clinical feature suggests the hepatic fibrosis process might contribute to cystic dilatation of the peribiliary glands. Enlarged peribiliary cysts can cause mechanical obstruction of the biliary tree and can also harbor a dysplastic change in the biliary epithelium, which may be induced by repeti- tive injury and regeneration of cholangiocytes. ${ }^{18-20}$

Biliary preneoplastic lesions, which are reported in peribiliary cysts, are biliary intraepithelial neoplasms and intraductal tubulopapillary neoplasms. These entities are well-known precursor lesions of extrahepatic cholangiocarcinoma or pancreatic adenocarcinoma, and they seem to be a counterpart of large duct type ICC. Chronic biliary diseases, such as cholelithiasis, parasitic infections or primary sclerosing cholangitis, are well-known risk factors for extrahepatic or peribilar cholangiocarcinoma. The general histopathologic phenotype of extrahepatic cholangiocarcinomas is a mucin producing tubular adenocarcinoma or an intestinal type adenocarcinoma. A peribiliary cyst may be a potential precursor lesion that can explain the occurrence of large duct type ICC in chronic liver disease patients without chronic biliary disease. Ambiguous histologic features of peribiliay glands between large and small bile ducts may explain the occurrence of intraductal tubulopapillary neoplasms of bile ducts, which is similar to intraductul tubulopapillry neoplasms of the pancreas.

\section{DIFFERENTIAL DIAGNOSIS OF BENIGN BILE DUCTS WITH DYSPLASIA}

Histopathologic features of the normal bile duct epithelium reacting to injury and regeneration are too wide and diverse to differentiate the dysplastic epithelium from the non-neoplastic epithelium.

However, protein or genetic alterations, which can be practically used in the differentiation of dysplastic cholangiocytes and reactive changes, were mainly reported in extrahepatic bile duct lesions, such as the common bile duct, distal bile duct, or gallbladder. ${ }^{21-23}$ Alteration of $\mathrm{p} 53$ or K-ras mutations were generally reported in large bile duct type cholangiocarcinomas; however, peripheral ICC had a different mutation phenotype from the low K-ras mutation and high IDH1 or IDH2 mutations. ${ }^{24,25}$ Histologic features implying an invasive phenotype or high proliferative activity in cells, such as an irregularity of the glandular border, fusion of glands, stratification of epithelial cells, pleomorphisms of cholangiocytes, hyperchomasia of nuclei, or necrotic debri in the glandular lumen, are also identified in exaggerated VMCs, ductular reactions, or bile duct adenomas. Further studies are required for evaluating diagnostic biomarkers. 


\section{SUMMARY}

Benign intrahepatic bile duct lesions, which have been regarded as reactive changes or remnants of developmental anomalies in the past, have been noted to have potential for evolving into precursor lesions of intrahepatic cholangiocarcinomas. Although these lesions have histopathological features overlapping with those of subtypes of ICC, confirmation as precursor lesions requires more evidence based on the large scale epidemiologic data supporting the association of VMC with increased incidence of ICC. More extensive studies for evaluating alterations of molecular or genetic markers are needed to understand the mechanism of oncogenesis and to make a precise diagnosis.

\section{Conflicts of Interest}

The author has no conflicts to disclose.

\section{REFERENCES}

1. Nakanuma Y, Tsutsui A, Ren XS, Harada K, Sato Y, Sasaki M. What are the precursor and early lesions of peripheral intrahepatic cholangiocarcinoma? Int J Hepatol 2014;2014:805973.

2. Aishima S, Oda Y. Pathogenesis and classification of intrahepatic cholangiocarcinoma: different characters of perihilar large duct type versus peripheral small duct type. J Hepatobiliary Pancreat Sci 2015;22:94-100.

3. Nakanuma Y. A novel approach to biliary tract pathology based on similarities to pancreatic counterparts: is the biliary tract an incomplete pancreas? Pathol Int 2010;60:419-429.

4. Terada T, Nakanuma Y. Development of human intrahepatic peribiliary glands. Histological, keratin immunohistochemical, and mucus histochemical analyses. Lab Invest 1993;68:261-269.

5. Terada T, Nakanuma Y, Kakita A. Pathologic observations of intrahepatic peribiliary glands in 1000 consecutive autopsy livers. Heterotopic pancreas in the liver. Gastroenterology 1990;98:1333-1337.

6. Wills ES, Roepman R, Drenth JP. Polycystic liver disease: ductal plate malformation and the primary cilium. Trends Mol Med 2014;20:261270.

7. Kida T, Nakanuma Y, Terada T. Cystic dilatation of peribiliary glands in livers with adult polycystic disease and livers with solitary nonparasitic cysts: an autopsy study. Hepatology 1992;16:334-340.

8. Choe JY, Kim H. Intrahepatic cholangiocarcinoma with predominant ductal plate malformation pattern. Clin Mol Hepatol 2014;20:214217.

9. Song JS, Lee YJ, Kim KW, Huh J, Jang SJ, Yu E. Cholangiocarcinoma arising in von Meyenburg complexes: report of four cases. Pathol Int
2008;58:503-512.

10. Jain D, Sarode VR, Abdul-Karim FW, Homer R, Robert ME. Evidence for the neoplastic transformation of Von-Meyenburg complexes. Am J Surg Pathol 2000;24:1131-1139.

11. Pinho AC, Melo RB, Oliveira M, Almeida M, Lopes J, Graca L, et al. Adenoma-carcinoma sequence in intrahepatic cholangiocarcinoma. Int J Surg Case Rep 2012;3:131-133.

12. Hasebe T, Sakamoto M, Mukai K, Kawano N, Konishi M, Ryu M, et al. Cholangiocarcinoma arising in bile duct adenoma with focal area of bile duct hamartoma. Virchows Arch 1995;426:209-213.

13. Theise ND, Nakashima O, Park YN, Nakanuma Y, Bosman FT, Garneiro $\mathrm{F}$, et al. WHO classification of tumours of the digestive system, 4th Ed: Lyon: IARC Press, 2010;205-227.

14. Tsui WM, Loo KT, Chow LT, Tse CC. Biliary adenofibroma. A heretofore unrecognized benign biliary tumor of the liver. Am J Surg Pathol 1993;17:186-192.

15. Godambe A, Brunt EM, Fulling KH, Reza Kermanshahi T. Biliary adenofibroma with invasive carcinoma: case report and review of the literature. Case Rep Pathol 2016;2016:8068513.

16. Nakanuma Y, Kurumaya H, Ohta G. Multiple cysts in the hepatic hilum and their pathogenesis. A suggestion of periductal gland origin. Virchows Arch A Pathol Anat Histopathol 1984;404:341-350.

17. Kim HJ, Kim CY, Hur YH, Kim JC, Cho CK, Kim HJ. Peribiliary cysts developed in normal underlying liver: report of a case. Korean J Hepatobiliary Pancreat Surg 2013;17:131-134.

18. Umemura A, Suto T, Sasaki A, Nitta H, Nakamura S, Endo F, et al. Pure laparoscopic left hemihepatectomy for hepatic peribiliary cysts with biliary intraepithelial neoplasia. Case Rep Surg 2016;2016:7236427.

19. Kai K, Eguchi Y, Kumagai T, Sugita Y, Tokunaga O. An autopsy case of obstructive jaundice due to hepatic multiple peribiliary cysts accompanying hepatolithiasis. Hepatol Res 2008;38:211-216.

20. Zen $Y$, Amarapurkar AD, Portmann BC. Intraductal tubulopapillary neoplasm of the bile duct: potential origin from peribiliary cysts. Hum Pathol 2012;43:440-445.

21. Aishima S, Iguchi T, Fujita N, Taketomi A, Maehara Y, Tsuneyoshi $M$, et al. Histological and immunohistological findings in biliary intraepithelial neoplasia arising from a background of chronic biliary disease compared with liver cirrhosis of non-biliary aetiology. Histopathology 2011;59:867-875.

22. Walter D, Herrmann E, Winkelmann R, Albert JG, Liese J, Schnitzbauer $A$, et al. Role of CD15 expression in dysplastic and neoplastic tissue of the bile duct - a potential novel tool for differential diagnosis of indeterminate biliary stricture. Histopathology $2016 \mathrm{Jul} 21$. [Epub ahead of print]

23. Nakanishi Y, Zen Y, Kondo S, Itoh T, Itatsu K, Nakanuma Y. Expression of cell cycle-related molecules in biliary premalignant lesions: biliary intraepithelial neoplasia and biliary intraductal papillary neo- 
Kyoung-Bun Lee

Histopathology of intrahepatic benign bile duct lesion

plasm. Hum Pathol 2008;39:1153-1161.

24. Liau JY, Tsai JH, Yuan RH, Chang CN, Lee HJ, Jeng YM. Morphological subclassification of intrahepatic cholangiocarcinoma: etiological, clinicopathological, and molecular features. Mod Pathol 2014;27:1163-1173.
25. Yu TH, Yuan RH, Chen YL, Yang WC, Hsu HC, Jeng YM. Viral hepatitis is associated with intrahepatic cholangiocarcinoma with cholangiolar differentiation and $\mathrm{N}$-cadherin expression. Mod Pathol 2011;24:810-819. 